\title{
Co-occurrence of nonsense mutations in MSH6 and MSH2 in Lynch syndrome families evidencing that not all truncating mutations are equal
}

\author{
Carla Pinto ${ }^{1}$, Manuela Pinheiro ${ }^{1}$, Ana Peixoto ${ }^{1}$, Catarina Santos ${ }^{1}$, Isabel Veiga ${ }^{1}$, Patrícia Rocha ${ }^{1}$, Pedro Pinto ${ }^{1}$, \\ Paula Lopes $^{2}$, Manuela Baptista ${ }^{3}$, Rui Henrique ${ }^{2,4}$ and Manuel R Teixeira ${ }^{1,4}$
}

The majority of pathogenic mismatch repair (MMR) gene mutations detected in Lynch syndrome patients are truncating (frameshift or nonsense). However, the classification of terminal truncating mutations is sometimes difficult and predictive testing based on non-deleterious variants can have very serious consequences. Here, we report eight probands that have two germline nonsense mutations, namely MSH6 c.1030C > T, p.(GIn344Ter) and MSH2 c.2785C > T, p.(Arg929Ter), and one additional patient who presented only the MSH2 mutation previously reported as deleterious. The novel MSH6 truncating mutation was classified as deleterious, as it is predicted to encode a protein with loss of 1017 amino acid residues.

The MSH2 mutation, which is expected to encode a protein lacking six amino acid residues, was considered a variant of unknown significance. Five tumors of the eight double-mutant individuals had normal MSH2 expression, whereas MSH6 immunoexpression was lost in all evaluable cases. None of the variants were detected in normal controls or associated with other MMR germline mutations in our series. This study emphasizes that not all truncating mutations are equal and that one must be cautious in the interpretation of the presumed deleterious effect of terminal frameshift or nonsense mutations.

Journal of Human Genetics (2016) 61, 151-156; doi:10.1038/jhg.2015.124; published online 8 October 2015

\section{INTRODUCTION}

Lynch syndrome, also known as Hereditary Nonpolyposis Colorectal Cancer (MIM\# 120435), is the most common hereditary colon cancer syndrome, representing $2-5 \%$ of all colorectal cancer (CRC), including a large proportion of those with a young age of diagnosis. ${ }^{1-3}$ Germline mutations in mismatch repair (MMR) genes (MLH1, MIM\# 120436; MSH2, MIM\# 609309; MSH6, MIM\# 600678; and PMS2, MIM\# 600259) are the major causes of Lynch syndrome, which is a highly penetrant autosomal dominant disease characterized by increased risk for CRC and extracolonic tumors of the endometrium, stomach, small bowel, ureter, renal pelvis, ovary and hepatobiliary tract. ${ }^{4}$ The vast majority of Lynch syndrome-associated tumors presents microsatellite instability due to their DNA MMR deficiency. In addition, microsatellite instability tumors can be identified by abnormal MMR immunohistochemical staining as the MMR gene mutated in the germline usually loses the wild-type allele in the tumor cells. 5

The detection of pathogenic MMR mutations in patients suspected of having Lynch syndrome confirms the diagnosis and allows genetic counseling and predictive testing in their relatives. ${ }^{6}$ About $90 \%$ of germline mutations in Lynch syndrome are found in MLH1 and MSH2, 7\% in MSH6 and 1\% in PMS2. ${ }^{7}$ The germline mutations in MLH1 or MSH2 are usually associated with clinically recognizable
Lynch syndrome families, whereas mutations in the MSH6 and PMS2 genes appear to be more associated with atypical Lynch syndrome. ${ }^{8-10}$ As molecular characterization of Lynch syndrome was established, the identification of gene carriers has become a critical issue with important clinical implications for cancer surveillance, incidence and mortality. ${ }^{11}$ The identification of patients with Lynch syndrome can be facilitated in populations with founder mutations by targeting the mutational analysis to specific gene regions as a first step, improving the cost effectiveness of the genetic testing strategy. ${ }^{12-14}$

The majority of MMR gene variants that are detected in Lynch syndrome patients are truncating mutations, which are generally classified as pathogenic. However, there are examples in the literature that terminal nonsense mutations may be polymorphisms with no major functional consequences for the encoding protein, as is the case of the terminal BRCA2 mutation c.9976A $>$ T, p.(Lys3326Ter). ${ }^{15}$ The classification of terminal truncating mutations may therefore be difficult and could have very serious consequences for cancer families if a non-deleterious variant is used for predictive genetic testing. We here present eight families that have two germline nonsense mutations, one novel mutation in MSH6 exon 4 and a MSH2 exon 16 mutation previously reported as deleterious.

${ }^{1}$ Department of Genetics, Portuguese Oncology Institute, Porto, Portugal; ${ }^{2}$ Department of Pathology, Portuguese Oncology Institute, Porto, Portugal; ${ }^{3}$ Department of Surgery, São João Hospital, Porto, Portugal and ${ }^{4}$ Institute of Biomedical Sciences (ICBAS), University of Porto, Porto, Portugal

Correspondence: Professor MR Teixeira, Department of Genetics, Portuguese Oncology Institute, Rua Dr. António Bernardino de Almeida, S/N, Porto 4200-072, Portugal.

E-mail: manuel.teixeira@ipoporto.min-saude.pt

Received 25 June 2015; revised 30 July 2015; accepted 11 September 2015; published online 8 October 2015 


\section{MATERIALS AND METHODS}

\section{Patients, samples and DNA extraction}

This study includes eight Lynch syndrome families presenting two concomitant truncating mutations in the MSH6 and MSH2 genes, from a total series of 114 families with pathogenic MLH1, MSH2, MSH6 or PMS2 germline mutations (data not shown). Seven of these index patients were identified by routine genetic diagnosis during the period of 1997-2014 at the Genetics Department of the Portuguese Oncology Institute, Porto, Portugal, after genetic counseling and informed consent. The remaining index patient was initially sent to us as a familial case belonging to a family with the $\mathrm{MSH} 2$ mutation identified in France. In addition to these eight families, we also identified one family that only presented the $\mathrm{MSH} 2$ germline mutation. Seven families were followed up at the Portuguese Oncology Institute and two at São João Hospital. Three families fulfilled the Amsterdam criteria, whereas the remaining presented the Bethesda criteria for genetic testing (Table 1 and Figure 1). ${ }^{16,17}$ After written informed consent, DNA was isolated from peripheral blood samples from the nine index individuals and subsequently from 16 family members, using standard procedures. The geographic origin of these families was inferred from the birthplace of the oldest carrier or of the oldest affected family member most likely to be a carrier.

\section{Screening for MMR germline alterations}

Genomic DNA from the eight patients was screened for MMR germline mutations. MSH2 and MSH6 coding exons and flanking regions were studied by denaturing gradient gel electrophoresis using primers and conditions as described by $\mathrm{Wu}$ and co-workers and Ingeny (Goes, The Netherlands) and/or by direct sequencing in an ABI PRISM 3500 automatic sequencer using Big Dye Terminator Chemistry (Applied Biosystems, Foster City, CA, USA), according to the manufacturer's recommendations. ${ }^{18}$ Whenever necessary, MSH6 exon 7 was re-sequenced using a different set of primers to exclude or confirm the presence of a polymorphism at the initial primer annealing site. ${ }^{19} \mathrm{MSH} 2$ and MSH6 exonic rearrangements were screened by multiplex ligation-dependent probe amplification, according to the SALSA MLPA P003 MLH1/MSH2 and P072 MSH6 kits (MRC-Holland, Amsterdam, The Netherlands) instructions. The variants described are according to the Human Genome Variation Society guidelines and we used the LRG_218 (NM_000251.2) for the MSH2 gene and the LRG_219 (NM_000179.2) for the MSH6 gene. Relatives were screened for the MMR germline mutations identified in the index patients by direct sequencing. In addition, KASPar SNP genotyping (LGC, Herts, UK) was used to screen the MSH2 and MSH6 mutations in 100 controls from the blood donors bank of the Portuguese Oncology Institute, Porto.

Table 1 Clinical data of the families with germline $\mathrm{MSH} 6$ and $\mathrm{MSH} 2$ mutations (families 1 to 8 ) or with only the $M S H 2$ variant (family 9)

\begin{tabular}{|c|c|c|c|}
\hline Family & Criteria & $\begin{array}{l}\text { Index cancer } \\
\text { (age) }\end{array}$ & Family history (age) \\
\hline 1 & Amsterdam & $C A(26)^{a}$ & EC (50), EC (49); EC (59); CRC (53) \\
\hline 2 & Bethesda & CRC (38) & CRC (70) \\
\hline 3 & Amsterdam & CA (31) & $\begin{array}{l}\mathrm{CRC}(70) ; \mathrm{CRC}(58) ; \mathrm{CRC} \text { (70); CRC (40); } \\
\text { IBL (34) }\end{array}$ \\
\hline 4 & Bethesda & CRC (48) & CRC (32); CRC (65) \\
\hline 5 & Bethesda & CRC (40) & CRC (49); PP (55) \\
\hline 6 & Amsterdam & CRC (47) & $\begin{array}{l}\text { CRC (45); CRC (67); BC (49); BT (56); EC } \\
\text { (42)+PaC (?); CRC (67); LC (70) }\end{array}$ \\
\hline 7 & Bethesda & OC (46) & CRC (37) \\
\hline 8 & Bethesda & EC (47) & EC (44); CRC (47) \\
\hline 9 & Bethesda & CRC (36) & No family history \\
\hline
\end{tabular}

Abbreviations: BC, breast cancer; BT, brain tumor; CA, colon adenoma; CRC, colorectal cancer; $\mathrm{EC}$, endometrial cancer; IBL, intestinal Burkitt lymphoma; LC, lung cancer; OC, ovarian cancer; $\mathrm{PaC}$, pancreatic cancer; PP, polyposis.

aPatient initially studied as a familial case belonging to a family with the MSH2 mutation identified in France.
MMR immunohistochemical analysis

Assessment of MLH1, MSH2, MSH6 and PMS2 immunoexpression was performed as previously described in the eight tumors from the patients harboring the two truncating mutations in MSH2 and MSH6 (the tumor from the patient with isolated $\mathrm{MSH} 2$ mutation was not available). ${ }^{20}$

\section{Microsatellite typing and haplotype analysis}

A total of 9 probands and 16 family members were genotyped for a subset of 10 microsatellite markers (D2S391, D2S2227, Clen33, Clen30, Clen29, D2S2156, D2S123, Clen43, Clen44 and D2S378) flanking MSH6 and MSH2. The physical distances of the genetic markers were derived from the National Center for Biotechnology Information (NCBI) Map Viewer (http://www.ncbi.nlm.nih.gov/ projects/mapview/). The primer sequences for amplification of the markers D2S391, D2S123 and D2S378 were derived from the Human Genome database; primers for markers D2S2227 and D2S2156 were designed using the online Primer-BLAST tool (http://www.ncbi.nlm.nih.gov/tools/primer-blast/), and markers Clen33, Clen30, Clen29, Clen43 and Clen44 were derived from Clendenning and co-workers. ${ }^{21}$ All 10 markers were assayed by PCR using fluorescently end-labeled primers. PCR products were run on an ABI PRISM 310 Genetic Analyzer together with the fluorescence-labeled DNA fragment size standard TAMRA (Applied Biosystems). Haplotype construction was performed manually based on the genotypes obtained from index cases and family members.

\section{Statistical analysis}

Comparison of mean age of CRC diagnosis was performed by Mann-Whitney test and $P$-values $<0.05$ were considered statistically significant.

\section{RESULTS}

\section{Mutation analyses}

Analysis of the constitutional DNA by denaturing gradient gel electrophoresis and Sanger sequencing in eight index cases initially studied in Porto revealed, in seven of them, two germline mutations, the MSH6 exon 4 c.1030C > T, p.(Gln344Ter) and the MSH2 exon 16 c.2785C > T, p.(Arg929Ter), whereas the eighth presented only the latter (Figure 2 and Table 2). In addition, one individual with a colon adenoma referred to our institution as a familial patient belonging to a Lynch syndrome family with the $\mathrm{MSH} 2$ mutation c.2785C $>\mathrm{T}$, p.(Arg929Ter), detected in a relative living in France, was included in this study. Given the co-occurrence of this $\mathrm{MSH} 2$ mutation with the MSH6 c.1030C > T mutation in seven of our families, both mutations were screened, and we observed that this patient also presented the two variants (Table 2). Furthermore, of the 16 family members studied, 5 were wild-type and 11 presented the two mutations concomitantly. Moreover, the MSH6 c.1030C $>\mathrm{T}$ and $\mathrm{MSH} 2$ c. $2785 \mathrm{C}>\mathrm{T}$ mutations were not found in the 100 healthy controls screened, nor in the remaining families tested by our group (251 probands tested for MSH2 and 118 probands tested for MSH6 germline mutations; data not shown).

\section{Clinical and phenotype analyses}

Three of the eight double-mutant families presented Amsterdam criteria for Lynch syndrome genetic analysis and three of these presented endometrial cancer in their family history (Table 1). The proband presenting only the MSH2 mutation had no family history of cancer (Table 1). The mean age of CRC onset in the double-mutant families was 52.7 years, which is closer to what has been reported for MSH6 mutated families, but does not significantly differ from that of the remaining Lynch syndrome families with pathogenic mutations studied by us (43.7 for MLH1, 44.2 for MSH2 and 43.5 for MSH6 mutation carriers; $P=0.992, P=0.992$ and $P=0.9522$, respectively). 
a

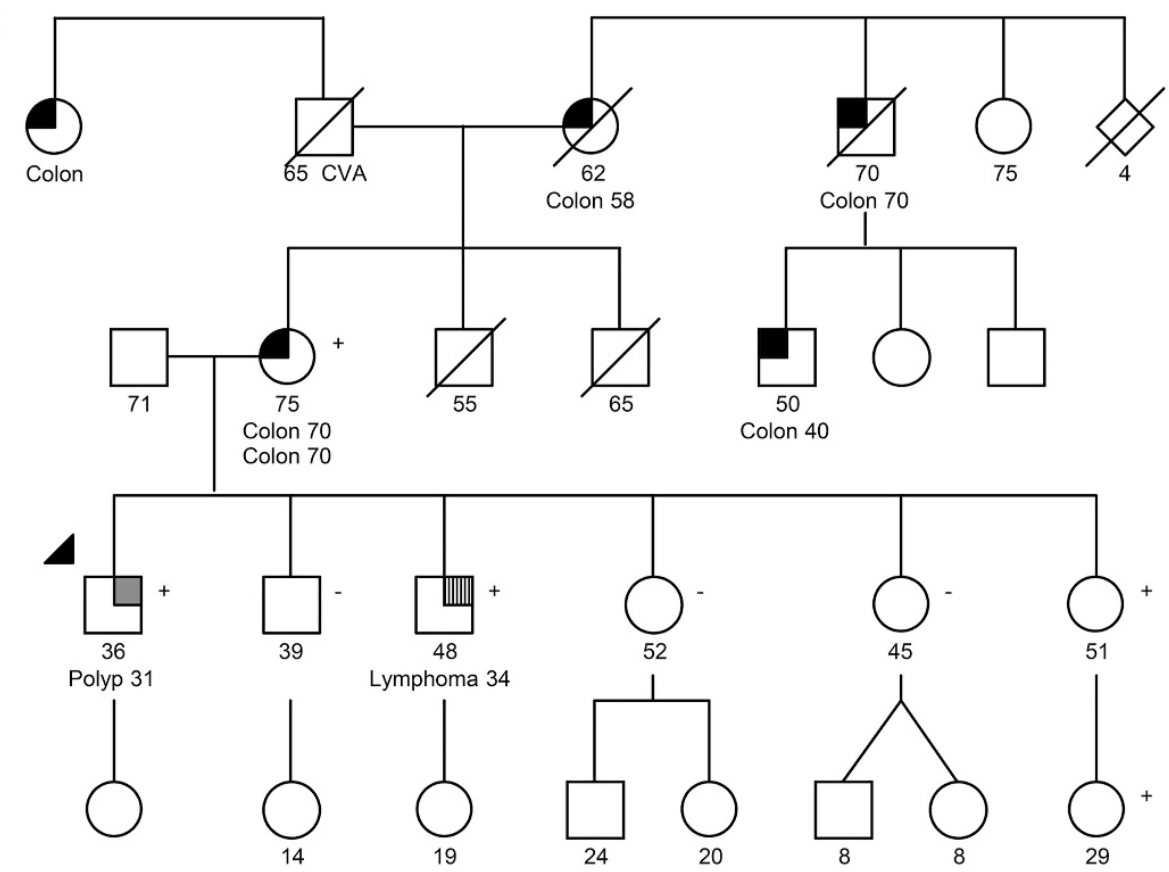

b

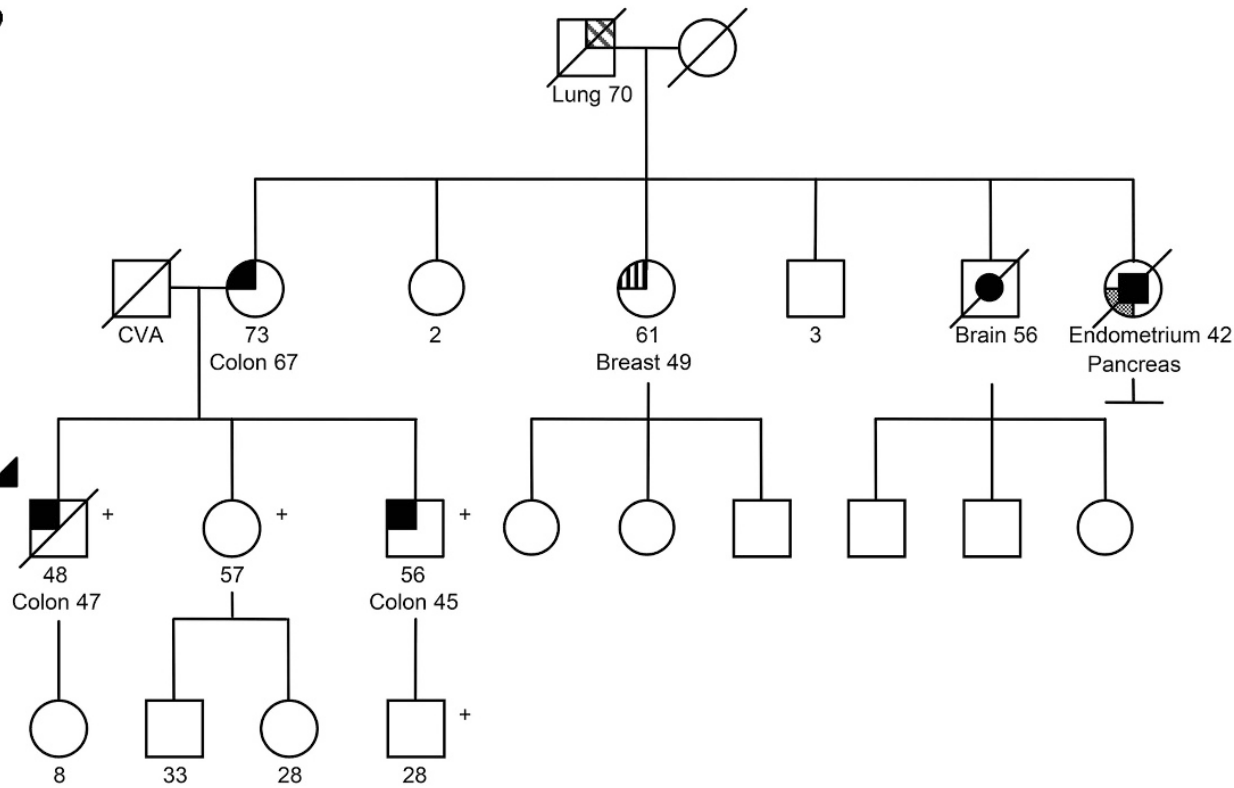

Figure 1 Two examples of pedigrees having co-occurrence of both MSH6 and MSH2 nonsense mutations: (a) family number 3; (b) family number 6 .

\section{Immunohistochemistry}

Immunohistochemistry analysis of tumor tissue revealed loss of MSH6 protein immunoexpression in seven of the eight double-mutant families, whereas the analysis of the eighth was not interpretable (due to the absence of MSH6 immunoreactivity also in the adjacent normal tissue). Regarding the MSH2 protein, five of these families presented normal expression, one presented decreased expression, and in two there was complete loss of expression in concomitance with the loss of MSH6 expression. All tumors presented normal MLH1 and PMS2 protein expression (Table 2 and Figure 3).

\section{Haplotype analysis}

Microsatellite haplotypes for all markers were phased in two out of the eight double-mutant Lynch syndrome families analyzed and two different haplotypes were observed. However, these two haplotypes present a conserved region of $\sim 1.5 \mathrm{Mb}$ encompassing the most telomeric markers (from the MSH6 gene to the D2S391 microsatellite marker). The remaining six double-mutant families and the index patient with only the MSH2 mutation presented unphased haplotypes, but harbored alleles consistent with the conserved region (Table 3).

All families presented in this study were apparently unrelated, but were originated from a restricted area, north to the city of Porto.

\section{DISCUSSION}

Molecular characterization of Lynch syndrome families is a fundamental step for their adequate clinical management. Knowing the deleterious constitutional mutation in the proband makes possible the identification of the family members that have the same risk as the 
general population and the relatives at high risk of developing Lynch syndrome associated cancers, allowing for cost-effective screening and/or prophylactic measures in the latter.

We, here, describe a set of eight families presenting nonsense mutations in two different MMR genes, namely MSH6 and MSH2. As all probands and mutation carrier relatives presented the two mutations in concomitance and both $\mathrm{MSH} 2$ and $\mathrm{MSH} 6$ are located in chromosome 2, we can infer that the two mutations are in cis, being, therefore, transmitted together unless a recombinational event occurs in chromosome 2 between the two MMR genes. These mutations were not detected in the remaining families suspected of Lynch syndrome studied at our Department (251 probands tested for MSH2 and 118 probands tested for MSH6 germline mutations) nor in 100 normal controls. To the best of our knowledge, the MSH6 mutation
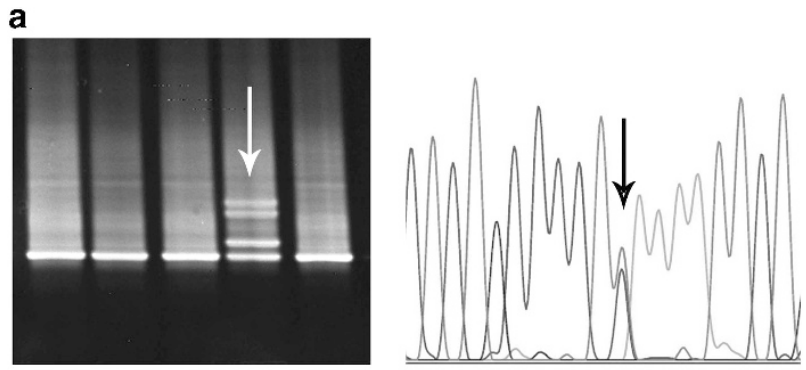

b
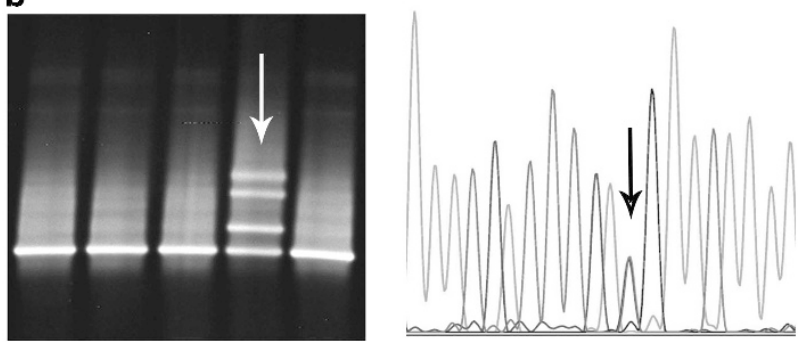

Figure 2 Germline mutations identified in $\mathrm{MSH} 6$ and $\mathrm{MSH}$ 2. (a) $\mathrm{MSH} 6$ exon 4c.1030C > T, p.(Gln344Ter). (b) MSH2 exon 16c.2785C >T, p. (Arg929Ter). Denaturing gradient gel electrophoresis (DGGE) gels are shown to the left, and sequence electropherograms are shown to the right; arrow indicates the mutations. A full color version of this figure is available at the Journal of Human Genetics journal online.
c.1030C > T, p.(Gln344Ter), has not previously been reported, and we classified it as deleterious because it is predicted to result in a protein lacking 1017 amino acid residues. The $\mathrm{MSH} 2$ mutation c.2785C > T, p.(Arg929Ter), on the other hand, occurs in the final 3' part of the gene, encoding a protein that is only six amino acids shorter and with no interruption of any known functional protein domain. This mutation has only been described previously in one individual who also presented the pathogenic c. $942+3 \mathrm{~A}>\mathrm{T}$ MSH 2 mutation, being classified as pathogenic in the InSiGHT database. ${ }^{22-24}$ Given that the MSH2 mutation c.2785C > T, p.(Arg929Ter), occurs in concomitance with a pathogenic mutation both in the eight families we here report and in the single family previously reported, that there is a lack of family history of cancer in the proband with only this $\mathrm{MSH} 2$ variant, together with the demonstration that $\mathrm{MSH} 2$ protein expression was normal in tumors of five double-mutant patients (Table 2), we consider this mutation as a variant of unknown significance, further studies being necessary to fully characterize this mutation. It should be noted that there are examples in the literature of nonsense variants classified as common polymorphisms, for instance, the BRCA2 c.9976A > T, p.(Lys3326Ter), which occurs in the last exon of the $B R C A 2$ gene and is predicted to encode a functional, shorter protein lacking the last 93 amino acids. ${ }^{15}$

Our data does not totally exclude the remote hypothesis that the two nonsense mutations in $\mathrm{MSH} 6$ and $\mathrm{MSH} 2$ may act cooperatively to confer an increased CRC risk. For instance, Martinez and Kolodner proposed that weak $M M R$ gene alleles are capable of polygenic interactions with other MMR gene alleles and thereby rising cancer risk. ${ }^{25}$ On the other hand, it is widely described in the literature that MSH6 mutation carriers develop CRC at a significantly older age than reported for $\mathrm{MLH1}$ and $\mathrm{MSH} 2$ mutation carriers, and it is also associated with an increased risk of developing endometrial carcinoma. ${ }^{10,26}$ The double-mutant families we, here, present have no atypical clinical features: the mean age of CRC diagnosis in the eight families (52.7 years) was higher than that of the MSH2 (44.2 years) or the MSH6 (43.5 years) mutation carrier families studied at our institution (although the difference is not statistically significant) and two families presented endometrial carcinoma. These data are consistent with the phenotype classically associated with MSH6 mutation carrier families, suggesting that the MSH2 variant does not increase the cancer risk in these families, supporting the idea that the pathogenicity of this mutation is uncertain.

Table 2 Molecular and immunohistochemistry data of the families with germline MSH6 and/or MSH2 mutations

\begin{tabular}{|c|c|c|c|c|c|c|c|c|}
\hline \multirow[b]{2}{*}{ Family } & \multicolumn{2}{|c|}{ MSH6 } & \multicolumn{2}{|c|}{ MSH2 } & \multicolumn{4}{|c|}{$\mathrm{IHC}$} \\
\hline & Mutation $^{\mathrm{a}}$ & Protein effect & Mutation $^{\mathrm{b}}$ & Protein effect & $M L H 1$ & PMS2 & MSH2 & MSH6 \\
\hline $1^{c}$ & c. $1030 \mathrm{C}>\mathrm{T}$ & p.(GIn344Ter) & c. $2785 \mathrm{C}>\mathrm{T}$ & p.(Arg929Ter) & Normal & Normal & No expression & No expression \\
\hline 2 & c. $1030 \mathrm{C}>\mathrm{T}$ & p.(GIn344Ter) & c. $2785 \mathrm{C}>\mathrm{T}$ & p.(Arg929Ter) & Normal & Normal & Normal & No expression \\
\hline $3^{d}$ & c. $1030 \mathrm{C}>\mathrm{T}$ & p.(GIn344Ter) & c. $2785 \mathrm{C}>\mathrm{T}$ & p.(Arg929Ter) & Normal & Normal & Normal & No expression \\
\hline 4 & c. $1030 \mathrm{C}>\mathrm{T}$ & p.(GIn344Ter) & c. $2785 \mathrm{C}>\mathrm{T}$ & p.(Arg929Ter) & Normal & Normal & Normal & Not interpretable \\
\hline 5 & c. $1030 \mathrm{C}>\mathrm{T}$ & p.(GIn344Ter) & c. $2785 \mathrm{C}>\mathrm{T}$ & p.(Arg929Ter) & Normal & Normal & No expression & No expression \\
\hline $6^{d}$ & c. $1030 \mathrm{C}>\mathrm{T}$ & p.(GIn344Ter) & c. $2785 \mathrm{C}>\mathrm{T}$ & p.(Arg929Ter) & Normal & Normal & Normal & No expression \\
\hline 7 & c. $1030 \mathrm{C}>\mathrm{T}$ & p.(GIn344Ter) & c. $2785 \mathrm{C}>\mathrm{T}$ & p.(Arg929Ter) & Normal & Normal & Diminished expression & No expression \\
\hline 8 & c. $1030 \mathrm{C}>\mathrm{T}$ & p.(GIn344Ter) & c. $2785 \mathrm{C}>\mathrm{T}$ & p.(Arg929Ter) & Normal & Normal & Normal & No expression \\
\hline 9 & Normal & Normal & c. $2785 \mathrm{C}>\mathrm{T}$ & p.(Arg929Ter) & NA & NA & NA & NA \\
\hline
\end{tabular}

Abbreviations: IHC, immunohistochemistry; MMR, mismatch repair; NA, tumor not available.

aAccording to NM_000179.2, nucleotide numbering starts with the A of the start codon.

bAccording to NM_000251.1, nucleotide numbering starts with the A of the start codon.

${ }^{c}$ MMR immunohistochemical analysis performed in the adenoma of the index patient.

${ }^{\mathrm{d}} \mathrm{MMR}$ immunohistochemical analysis performed in one carcinoma from a relative of the index case. 
a

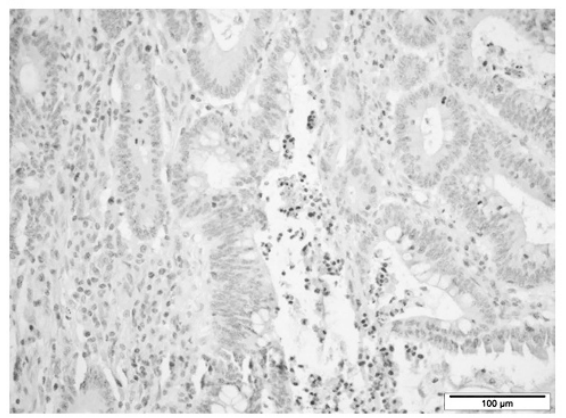

c

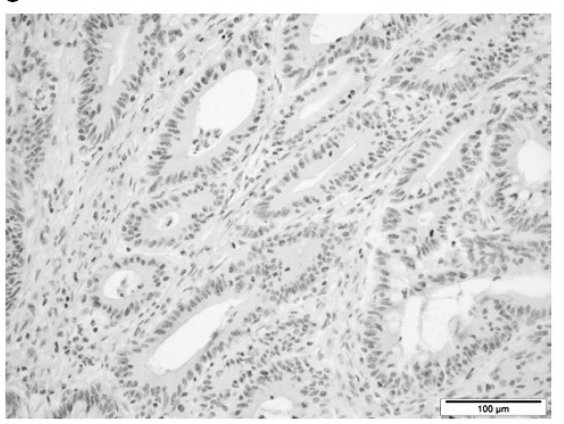

b

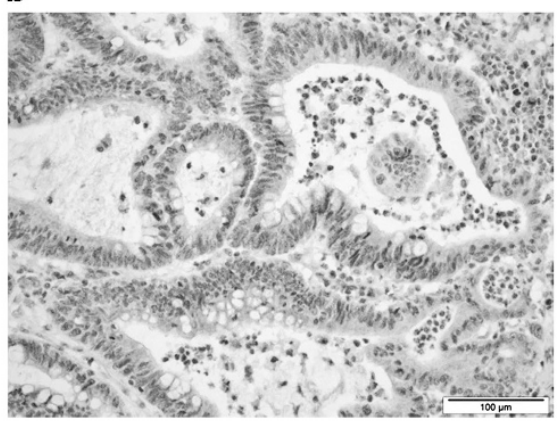

d

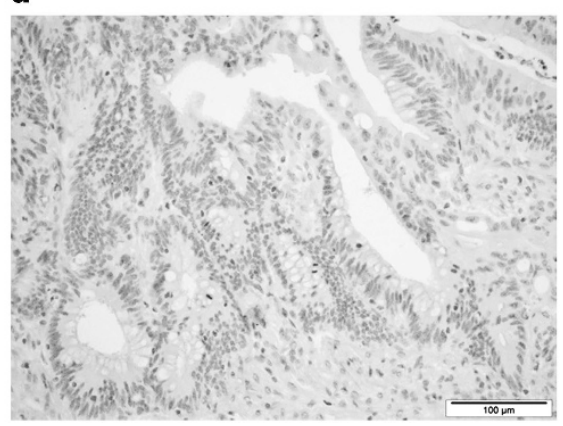

Figure 3 Immunohistochemistry analyses of a tumor from a carrier of both MSH6 and MSH2 truncating variants, showing loss of expression of MSH6 (a) and normal expression of MSH2 (b), MLH1 (c) and PMS2 (d). A full color version of this figure is available at the Journal of Human Genetics journal online.

Table 3 Microsatellite marker haplotypes of the Portuguese Lynch syndrome families presenting the germline $M$ SH6 and/or $M S H 2$ mutations

\begin{tabular}{|c|c|c|c|c|c|c|c|c|c|}
\hline \multirow[b]{2}{*}{ Marker } & \multicolumn{9}{|c|}{ Familya } \\
\hline & 3 & 5 & 2 & 6 & 4 & 7 & 8 & 1 & 9 \\
\hline D2S391 & 142 & 142 & 142 & 142 & 142 & 142 & $140 / 142$ & 142 & $142 / 146$ \\
\hline D2S2227 & 129 & 129 & 129 & $117 / 129$ & $117 / 129$ & $117 / 129$ & $117 / \mathbf{1 2 9}$ & $117 / 129$ & $117 / 129$ \\
\hline Clen33 & 162 & 162 & 162 & 162 & $158 / 162$ & $160 / 162$ & $158 / 162$ & $158 / 162$ & $158 / 162$ \\
\hline MSH6 & mut & mut & mut & mut & mut & mut & mut & mut & $w t$ \\
\hline Clen29 & 184 & 186 & 184 & 184 & $146 / 184$ & $146 / 184$ & $172 / 184$ & $172 / \mathbf{1 8 4}$ & $146 / 180$ \\
\hline D2S2156 & 123 & 123 & 123 & 123 & $119 / 125$ & 123 & 123 & $121 / 123$ & 123 \\
\hline D2S123 & 209 & 209 & 209 & 209 & 209 & 209 & 209 & 209/211 & $209 / 225$ \\
\hline Clen43 & 179 & 173 & $177 / \mathbf{1 7 9}$ & 179 & $159 / 177$ & $173 / 179$ & $169 / 179$ & $175 / 179$ & $173 / 179$ \\
\hline
\end{tabular}

Abbreviations: mut, mutated; wt, wild type.

aThe families are organized by a grade of informative haplotype. In the unphased haplotypes, the alleles that are consistent with the phased haplotypes (families 3 and 5 ) are indicated in bold.

The finding of double MSH2/MSH6 variants in eight families originated from a confined geographic area, north to the city of Porto, indicates that they might have a common ancestor. This is in accordance with the haplotype analysis showing that all families harbored alleles consistent with a conserved region. On the other hand, the available data does not allow us to infer the mechanism behind the co-occurrence of these two mutations in eight families and the presence of the isolated $\mathrm{MSH} 2$ variant in another family. Hypothetically, the mutation in the MSH6 gene could have occurred in a haplotype previously carrying the $\mathrm{MSH} 2$ variant. Alternatively, both could have occurred in the same haplotype of chromosome 2 and a subsequent recombination event could have separated the two variants. Finally, considering that the $\mathrm{MSH} 2$ mutation has been also described in one family outside Portugal, we cannot exclude that this mutation may have occurred independently due to a recurrent mutational event. Be that as it may, this study emphasizes that not all truncating mutations are equal and that one must be cautious in the interpretation of the presumed deleterious effect of terminal frameshift or nonsense mutations.

\section{CONFLICT OF INTEREST}

The authors declare no conflict of interest. 
1 Petersen, G. M., Brensinger, J. D., Johnson, K. A. \& Giardiello, F. M. Genetic testing and counseling for hereditary forms of colorectal cancer. Cancer 86, 2540-2550 (1999).

2 Umar, A., Risinger, J. I., Hawk, E. T. \& Barrett, J. C. Testing guidelines for hereditary non-polyposis colorectal cancer. Nat. Rev. Cancer 4, 153-158 (2004).

3 Hampel, H., Frankel, W. L., Martin, E., Arnold, M., Khanduja, K., Kuebler, P. et al. Feasibility of screening for Lynch syndrome among patients with colorectal cancer. J. Clin. Oncol. 26, 5783-5788 (2008)

4 Lynch, H. T. \& de la Chapelle, A. Hereditary colorectal cancer. N. Engl. J. Med. $\mathbf{3 4 8}$ 919-932 (2003)

5 Lynch, H. T., Boland, C. R., Gong, G., Shaw, T. G., Lynch, P. M., Fodde, R. et al. Phenotypic and genotypic heterogeneity in the Lynch syndrome: diagnostic, surveillance and management implications. Eur. J. Hum. Genet. 14, 390-402 (2006).

6 Ou, J., Niessen, R. C., Vonk, J., Westers, H., Hofstra, R. M. \& Sijmons, R. H. A database to support the interpretation of human mismatch repair gene variants. Hum. Mutat. 29, 1337-1341 (2008)

7 Peltomäki, P. \& Vasen, H. Mutations associated with HNPCC predisposition—update of ICG-HNPCC/INSiGHT mutation database. Dis. Markers 20, 269-276 (2004).

8 Sánchez de Abajo, A., de la Hoya, M., Tosar, A., Godino, J., Fernández, J. M., Asenjo, J. L. et al. Low prevalence of germline hMSH6 mutations in colorectal cancer families from Spain. World J. Gastroenterol. 11, 5770-5776 (2005).

9 Truninger, K., Menigatti, M., Luz, J., Russell, A., Haider, R., Gebbers, J. O. et al. Immunohistochemical analysis reveals high frequency of PMS2 defects in colorectal cancer. Gastroenterology 128, 1160-1171 (2005).

10 Boland, C. R., Koi, M., Chang, D. K. \& Carethers, J. M. The biochemical basis of microsatellite instability and abnormal immunohistochemistry and clinical behavior in Lynch syndrome: from bench to bedside. Fam. Cancer 7, 41-52 (2008).

11 Payá, A., Alenda, C., Pérez-Carbonell, L., Rojas, E., Soto, J. L., Guillén, C. et al. Utility of p16 immunohistochemistry for the identification of Lynch syndrome. Clin. Cancer Res. 15, 3156-3162 (2009).

12 de la Chapelle, A. Genetic predisposition to colorectal cancer. Nat. Rev. Cancer 4 , 769-780 (2004).

13 Pinheiro, M., Pinto, C., Peixoto, A., Veiga, I., Mesquita, B., Henrique, R. et al. A nove exonic rearrangement affecting MLH1 and the contiguous LRRFIP2 is a founder mutation in Portuguese Lynch syndrome families. Genet. Med. 13, 895-902 (2011).

14 Pinheiro, M., Pinto, C., Peixoto, A., Veiga, I., Mesquita, B., Henrique, R. et al. The MSH2 c.388_389del mutation shows a founder effect in Portuguese Lynch syndrome families. Clin. Genet. 84, 244-250 (2013).
15 Mazoyer, S., Dunning, A. M., Serova, O., Dearden, J., Puget, N., Healey, C. S. et al. A polymorphic stop codon in BRCA2. Nat. Genet. 14, 253-254 (1996).

16 Vasen, H. F., Watson, P., Mecklin, J. P. \& Lynch, H. T. New clinical criteria for hereditary nonpolyposis colorectal cancer (HNPCC, Lynch syndrome) proposed by the International Collaborative group on HNPCC. Gastroenterology 116, 1453-1456 (1999).

17 Umar, A., Boland, C. R., Terdiman, J. P., Syngal, S., de la Chapelle, A., Rüschoff, J. et al. Revised Bethesda Guidelines for hereditary nonpolyposis colorectal cancer (Lynch syndrome) and microsatellite instability. J. Natl Cancer Inst. 96, 261-268 (2004)

18 Wu, Y., Berends, M. J., Mensink, R. G., Kempinga, C., Sijmons, R. H., van Der Zee, A. G. et al. Association of hereditary nonpolyposis colorectal cancer-related tumors displaying low microsatellite instability with MSH6 germline mutations. Am. J. Hum. Genet. 65, 1291-1298 (1999).

19 Kolodner, R. D., Tytell, J. D., Schmeits, J. L., Kane, M. F., Gupta, R. D., Weger, J. et al. Germ-line msh6 mutations in colorectal cancer families. Cancer Res. 59, 5068-5074 (1999)

20 Pinheiro, M., Ahlquist, T., Danielsen, S. A., Lind, G. E., Veiga, I., Pinto, C. et al. Colorectal carcinomas with microsatellite instability display a different pattern of target gene mutations according to large bowel site of origin. BMC Cancer 10, 587 (2010).

21 Clendenning, M., Baze, M. E., Sun, S., Walsh, K., Liyanarachchi, S., Fix, D. et al. Origins and prevalence of the American Founder Mutation of MSH2. Cancer Res. 68, 2145-2153 (2008).

22 Valentin, M. D., da Silva, F. C., dos Santos, E. M., Lisboa, B. G., de Oliveira, L. P., Ferreira Fde, $\mathrm{O}$. et al. Characterization of germline mutations of MLH1 and MSH2 in unrelated south American suspected Lynch syndrome individuals. Fam. Cancer 10, 641-647 (2011)

23 InSiGHT (International Society for Gastrointestinal Hereditary Tumours) database. Available at http://insight-group.org/variants/database/. Accessed on 27 March 2015.

24 Thompson, B. A., Spurdle, A. B., Plazzer, J. P., Greenblatt, M. S., Akagi, K., Al-Mulla., F. et al. Application of a 5-tiered scheme for standardized classification of 2,360 unique mismatch repair gene variants in the InSiGHT locus-specific database. Nat. Genet. 46, 107-115 (2014).

25 Martinez, S. L. \& Kolodner, R. D. Functional analysis of human mismatch repair gene mutations identifies weak alleles and polymorphisms capable of polygenic interactions. Proc. Natl Acad. Sci. USA 107, 5070-5075 (2010).

26 Hendriks, Y. M., Wagner, A., Morreau, H., Menko, F., Stormorken, A., Quehenberger, F. et al. Cancer risk in hereditary nonpolyposis colorectal cancer due to MSH6 mutations: impact on counseling and surveillance. Gastroenterology 127, 17-25 (2004). 